\title{
Fidedignidade inter e intradias de um teste de potência muscular
}

\author{
Roberto Simão ${ }^{1}$, Walace Monteiro ${ }^{1,2}$ e Claudio Gil Soares de Araújo ${ }^{1,3}$
}

\section{RESUMO}

Com o envelhecimento há rápida e relevante perda de potência muscular (PM), prejudicando a autonomia e a qualidade de vida, tornando conveniente avaliar a PM. O objetivo do estudo foi determinar a fidedignidade inter e intradias de um teste simples de PM, realizado em uma carga previamente individualizada. Avaliamos 18 jovens adultos saudáveis, sendo 12 mulheres, inexperientes quanto a exercícios de fortalecimento. Determinou-se inicialmente 1RM com medida simultânea da velocidade e da potência $(F i$ trodyne, Bratislava), no exercício remada alta, até a altura mesoesternal na posição ortostática, obtendo-se ainda a carga na qual se conseguia a maior PM. Na semana seguinte, por cinco dias consecutivos, eles realizaram em quatro dias $2 \times 2$ repetições e em um dia $10 \times 2$ repetições (3s de intervalo entre as repetições), o mais rápido possível na fase concêntrica, com a carga de PM. Comparando os resultados pela ANOVA para medidas repetidas e teste de Bonferroni, verificou-se que a PM máxima não diferia - médias entre 262 e $267 \mathrm{~W}(\mathrm{p}=0,69)$. Para a variabilidade dos dados individuais, encontraram-se valores de 3 e $8 \%$, respectivamente, para o coeficiente de variação (CV) e para a média da variação dos resultados pela média dos indivíduos M-m/X. Nas dez séries consecutivas os valores ficaram entre 242 e $263 \mathrm{~W}$, somente identificando-se diferenças entre as séries 1 e 4 e 6 ( p < 0,05). Excluindo-se os dados da primeira série (cinco dos 18 avaliados tiveram valores excepcionalmente baixos nela), as diferenças desaparecem $(\mathrm{p}=0,13)$. Dados da variabilidade individual demonstraram 6 e $20 \%$, respectivamente, para CV e M-m/

\footnotetext{
1. Programa de Pós-Graduação em Educação Física/Universidade Gama Filho, Rio de Janeiro, RJ.

2. Laboratório de Fisiologia do Exercício/Nuicaf - Aeronáutica, Rio de Janeiro, RJ.

3. Clinimex - Clínica de Medicina do Exercício, Rio de Janeiro, RJ.

Recebido em: 17/5/2001.

Aceito em: 15/7/2001.
}

Endereço para correspondência:

Dr. Claudio Gil S. Araújo

Clínica de Medicina do Exercício - Clinimex

Rua Siqueira Campos, 93/101 - 22031-070 - Rio de Janeiro, RJ, Brasil.

E-mail: cgaraujo@iis.com.br

118
$\mathrm{X}$, nas dez séries consecutivas. Os autores concluem que a PM máxima obtida em um teste simples apresenta alta fidedignidade inter e intradias e variabilidade relativamente baixa, quando comparadas com outras mensurações freqüentemente usadas em ciências do exercício. Esse teste pode ser recomendado para medidas de PM em programas voltados para a saúde e a aptidão física, nos quais a utilização de materiais e protocolos mais sofisticados pode ser inapropriada ou inviável.

Palavras-chave: Potência muscular máxima. Teste de potência muscular. Força máxima. Treinamento contra resistência. Fortalecimento muscular. Fidedignidade inter e intradias.

\section{ABSTRACT}

\section{Inter and intraday reliability of a test of muscle power}

Aging induces fast and relevant decrements in muscle power (MP), reducing autonomy and quality of life. The authors' aim was to determine reliability data in a new, simple protocol for muscle power using a previously individualized load. The authors evaluated 18 healthy young adults (12 women), unaccustomed to strengthening exercises. Initially, 1-RM was determined with simultaneous measurement of velocity and MP (Fitrodyne, Bratislava) in an upright row exercise, performed up to mesosternal level in standing position, and measurement of load in which $M P$ was obtained. In the following week, they returned in five consecutive days to perform $2 \times 2$ reps in four days and in one day $10 \times 2$ reps ( $3 s$ interval between reps), using maximal velocity in the concentric phase. Comparing best results by repeated measures ANOVA-Bonferroni t-tests, PM did not differ - mean values ranging from 262 to $267 \mathrm{~W}$ ( $p$ $=.69$ ). Looking for individual data variability, values of 3 and $8 \%$ were found, respectively, for coefficient of variation $(\mathrm{CV})$ and for the mean range of scores divided by the mean value $(M-m / X)$ (maximum minus minimum score divided by the subject's mean value). Analyzing intraday reliability in the 10 consecutive sets - mean results (ranging from 242 to $263 \mathrm{~W}$ ) - significant differences were only found between set 1 and 4 and $6(p<.05)$. If set 1 data were excluded (5 of the 18 subjects performed very low values in this set), the differences disappeared $(p=.13)$. Individ- 
ual variability data showed 6 and 20\%, respectively, for $C V$ and $M-m / X$ in the 10 consecutive sets. The authors concluded that MP obtained in this simple test presents high intraday and interday reliability with relatively low variability, comparable to that often observed in other measurements usually used in exercise sciences. This test can be recommended for MP assessment in health and fitness facilities, where utilization of more sophisticated devices and protocols are either inappropriate or unsuitable.

Key words: Maximal muscle power. Muscle power test. Strength training. Resistance training. Inter and intraday reliability.

\section{INTRODUÇÃO}

Há muito tempo se discutem as definições e as aplicações da aptidão física ${ }^{1}$. A aptidão física relaciona-se com a saúde e com a performance, estando vários dos seus componentes especificamente ligados a cada uma dessas duas situações. Dentre os componentes da aptidão física relacionados à saúde, destacam-se a força e a potência musculares, relevantes para a qualidade de vida e a prescrição de exercícios em indivíduos sadios, bem como naquelas que apresentam necessidades específicas ${ }^{2,3}$.

Estudos sobre treinamento de força e potência são comuns na literatura científica ${ }^{4,5}$. Durante muito tempo priorizou-se o componente aeróbio da aptidão física; todavia, a partir dos anos 80, o treinamento de força começou a ser mais valorizado, sendo sua inclusão recomendada pelo Colégio Americano de Medicina do Esporte ${ }^{6}$. Programas visando seu desenvolvimento cresceram em popularidade, sendo hoje inegável sua presença em centros de saúde, clubes e academias.

Algumas definições de força e potência são freqüentemente contraditórias e confusas, dificultando o bom entendimento e a troca de experiências entre profissionais ${ }^{7}$. Entretanto, pelas leis que regem a física, a força é expressa como o produto da massa pela aceleração $(\mathrm{F}[\mathrm{N}]=\mathrm{m}[\mathrm{kg}])$. a $[\mathrm{m} / \mathrm{s}])$. Já a potência representa o trabalho mecânico desenvolvido sob uma série de condições $(1 \mathrm{~W}=$ trabalho realizado como $1 \mathrm{~J} / \mathrm{s}=$ torque de $1 \mathrm{~N} . \mathrm{m}$, acionando a velocidade de um radiano/s), podendo ser caracterizada da seguinte forma: $\left[\mathrm{P}(\mathrm{W})=\mathrm{F}(\mathrm{N}) \times \mathrm{V} \cdot\left(\mathrm{m} \cdot \mathrm{s}^{-1}\right)\right]$. Fugindo da problemática terminológica controversa (ex.: força explosiva, etc.), temos que a potência muscular é altamente dependente da força e ambas são importantes para performances desportivas e para o desempenho de atividades cotidianas ${ }^{4}$.

Posto isso, são necessárias formas simples de medidas que, além do baixo custo, apresentem resultados confiáveis. Dessa forma, o objetivo do presente estudo foi ava- liar a fidedignidade inter e intradias de um teste de potência muscular, através da utilização de um tensiômetro Fitrodyne (Bratislava, Eslováquia), no exercício remada em pé.

\section{MÉTODOS}

Foram estudados 18 indivíduos (12 mulheres) com idade entre 19 e 35 anos (28,7 $\pm 5,6$ anos), peso corporal entre 50 e $87 \mathrm{~kg}(67,4 \pm 11,4 \mathrm{~kg})$ e estatura entre 152 e $187 \mathrm{~cm}$ (168,4 \pm 9,4cm). Dos avaliados, 15 (dez mulheres e cinco homens) nunca haviam praticado exercícios de fortalecimento muscular e os outros três (duas mulheres e um homem) não participavam desse tipo de treinamento havia pelo menos um ano.

Todos os indivíduos estudados eram fisicamente ativos, já que praticavam exercícios físicos pelo menos três vezes semanais ${ }^{3,6}$. Antes da coleta de dados, todos os voluntários responderam negativamente aos itens do questionário PAR$\mathrm{Q}^{8}$ e assinaram um termo de consentimento. A coleta de dados constou das seguintes etapas: medidas do peso corporal, estatura e a aplicação de um teste de execução de movimentos completos na maior velocidade possível na remada em pé com aumento sucessivo de cargas, até a carga em que o indivíduo não conseguia completar o movimento. Dessa forma, determinou-se uma repetição máxima $(1 \mathrm{RM})^{9} \mathrm{e}$ a carga que gerava a maior potência muscular nesse exercício.

Objetivando reduzir a margem de erro no teste de $1 \mathrm{RM} \mathrm{e}$, posteriormente, na fidedignidade inter e intradias de um teste de potência muscular, adotaram-se as seguintes estratégias:

1) Instruções padronizadas foram oferecidas antes do teste, de modo que o avaliado estivesse ciente de toda a rotina que envolvia a coleta de dados.

2) O avaliado foi instruído sobre a técnica de execução do exercício, inclusive realizando-o algumas vezes sem carga, para reduzir um possível efeito do aprendizado nos escores obtidos. $\mathrm{O}$ avaliado realizava um número de repetições necessárias para sentir-se seguro na execução do exercício.

3) $\mathrm{O}$ avaliador estava atento quanto à posição adotada pelo praticante no momento da medida. Pequenas variações no posicionamento das articulações envolvidas no movimento podiam acionar outros músculos, levando a interpretações errôneas dos escores obtidos.

4) Os pesos e a barra de ferro utilizados no estudo foram checados em balança previamente calibrada.

5) Os testes foram sempre realizados nos mesmos horários para um dado indivíduo.

Para estabelecermos a carga que gerava a maior potência muscular e a carga máxima no teste de 1RM, utilizaram-se uma barra de $8 \mathrm{~kg}$ e pesos de 1, 2, 3, 4, 5 e $10 \mathrm{~kg}$. 


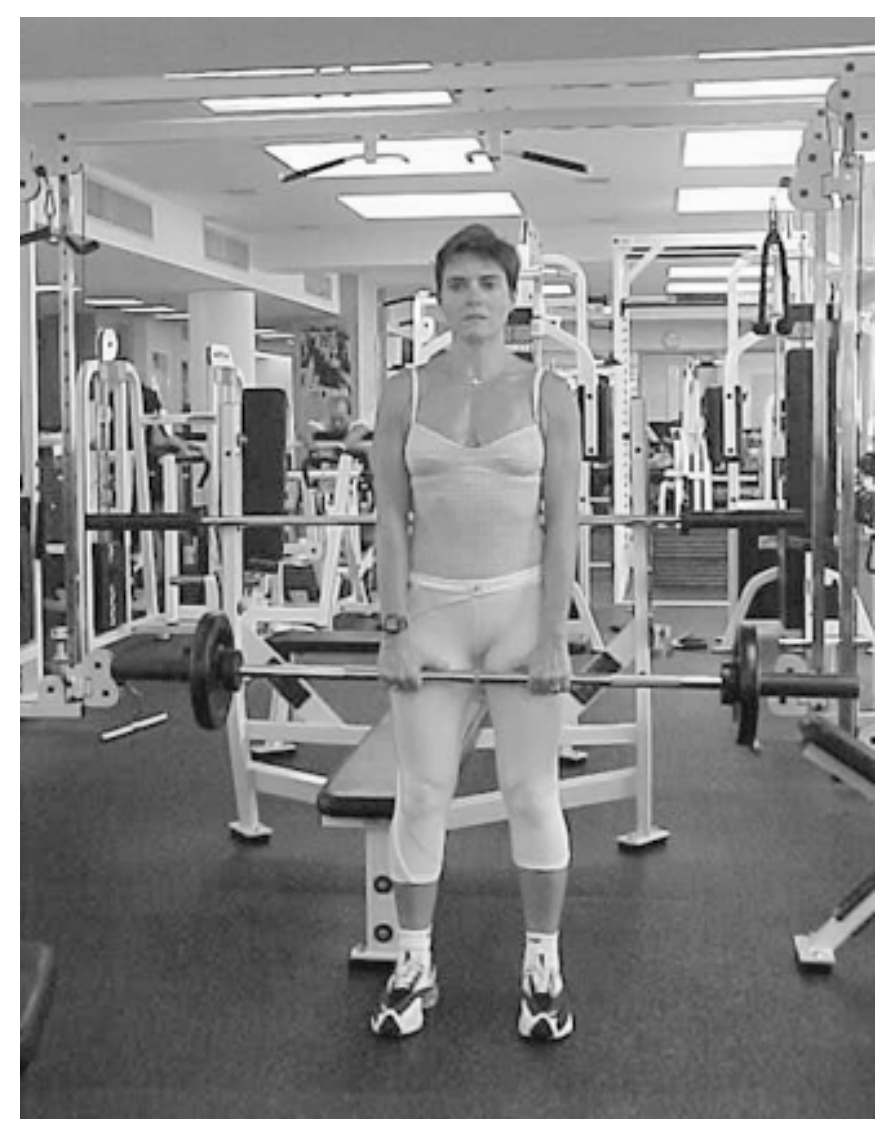

Fig. 1 - Posição inicial

Para a medida da potência muscular no exercício foi fixado no centro da barra um tensiômetro Fitrodyne (Bratislava, Eslováquia). Para cada execução na fase concêntrica, digitava-se a carga e o tensiômetro registrava a velocidade média alcançada e calculava a potência média em watts. Visando assegurar uma execução padronizada do exercício no teste de $1 \mathrm{RM}$ e, posteriormente, no procedimento da fidedignidade inter e intradias de um teste de potência muscular, estabeleceram-se as seguintes etapas de execução: a) posição inicial; b) fase ascendente da carga (fase concêntrica do movimento); c) fase descendente da carga (fase excêntrica do movimento).

a) Posição inicial - o indivíduo ficava de pé, pernas paralelas com um pequeno afastamento lateral, com os joelhos estendidos, quadris na posição anatômica, braços ao longo do corpo com as mãos segurando a barra e a cabeça posicionada com o plano de Frankfurt ${ }^{10}$. O posicionamento das mãos na barra para cada avaliado foi padronizado a partir do afastamento das mesmas em aproximadamente 20 a $25 \mathrm{~cm}$ (figura 1 ).

b) Fase ascendente - consistiu de abdução de ombros, concomitante à flexão dos cotovelos até o ponto mesoesternal para evitar qualquer risco de lesão ${ }^{11}$ (figura 2).

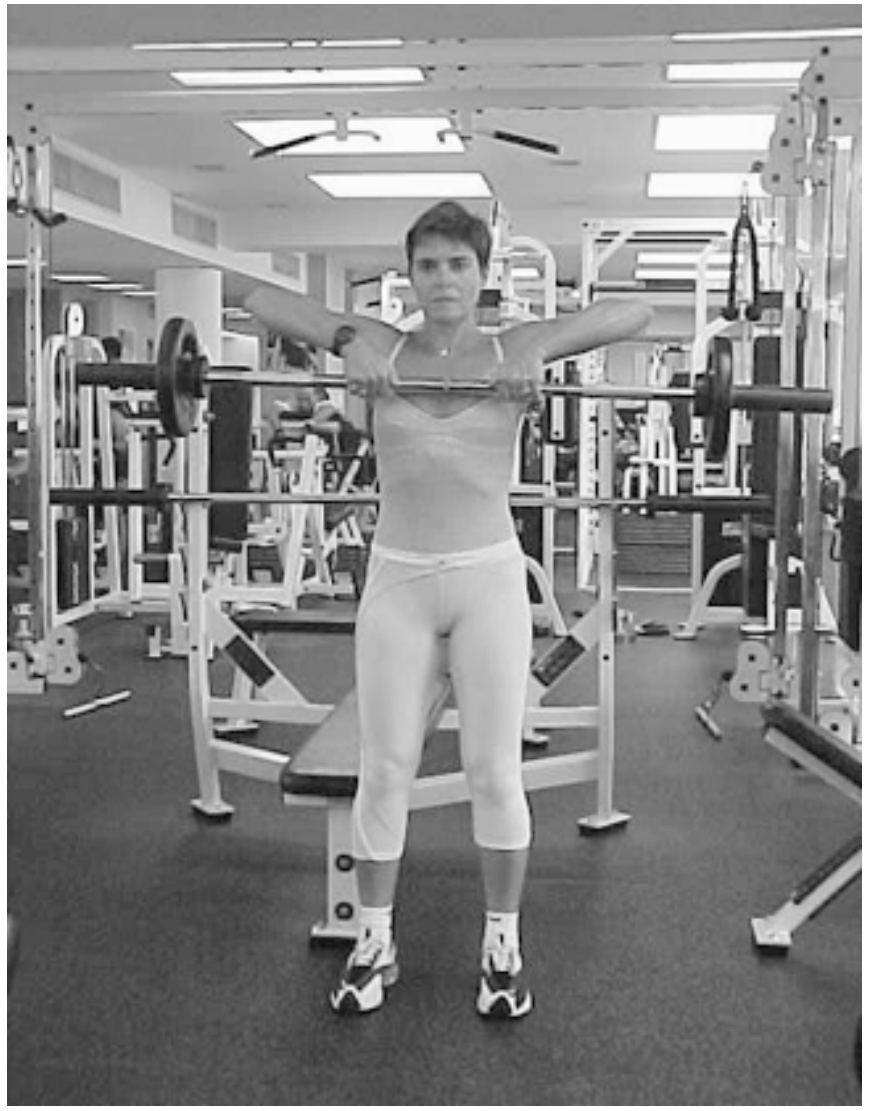

Fig. 2 - Fase ascendente

c) Fase descendente - a partir do final da fase ascendente, os cotovelos e os ombros voltam lentamente à posição inicial.

Ao iniciar o teste de 1RM, o avaliado realizava duas repetições, com três segundos de intervalo entre as repetições, imprimindo velocidade máxima na fase concêntrica do exercício. A maior potência muscular obtida das duas repetições mensuradas pelo Fitrodyne era considerada como a melhor. Seguiam-se incrementos progressivos de $2 \mathrm{~kg}$, a cada nova série de duas repetições, sendo dado um intervalo de um minuto entre cada série.

$\mathrm{O}$ procedimento do teste de $1 \mathrm{RM}$ tinha o propósito de obter duas cargas: a que gerava a maior potência muscular e a carga máxima. Quando o avaliado atingia a maior carga em um movimento correto, o teste era interrompido. Dessa forma, validou-se como carga máxima aquela obtida na última execução. Após obtida a carga que gerava o maior valor de potência muscular, tal valor foi utilizado para determinação da fidedignidade inter e intradias de um teste de potência muscular.

Finalmente, passou-se a realizar, durante cinco dias consecutivos, a mensuração da fidedignidade do teste. Para minimizar o efeito da habituação, adotou-se a técnica do 


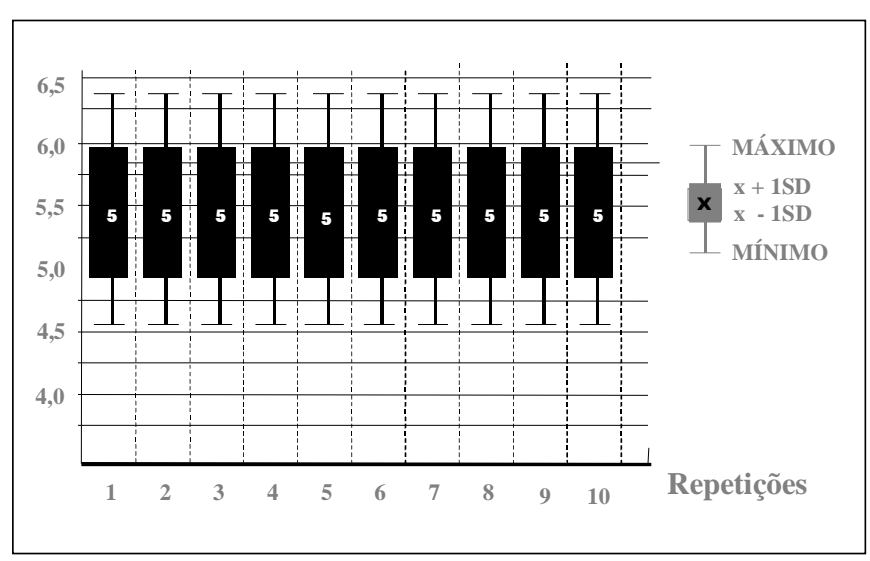

Fig. 3 - Potência muscular máxima intradias (logaritmo)

quadrado latino. Dos cinco dias de testes, em quatro realizaram-se duas séries de duas repetições, na maior velocidade possível, na fase concêntrica do exercício, considerando-se novamente a maior potência muscular em cada série. Em outro dia, de acordo com a alocação aleatória do quadrado latino, foram executadas dez séries de duas repetições, com o mesmo tempo de intervalo entre as repetições e séries. A melhor performance da potência muscular era utilizada conforme descrito anteriormente.

A comparação entre os resultados da potência muscular e da velocidade para a carga individualizada nos diferentes dias e nas dez séries do mesmo dia foi feita pela Anova para medidas repetidas e pelo teste de comparações múltiplas de Bonferroni para especificar quais medidas diferiam entre si, sendo adotado um nível de significância de $5 \%$. Considerando que a distribuição dos dados possuía alguma assimetria, repetiu-se a análise estatística com os dados submetidos à transformação logarítmica.

\section{RESULTADOS}

Com o intuito de evitar uma contaminação dos dados na análise da potência muscular máxima e velocidade, ao longo do mesmo dia, eliminou-se a primeira execução de cada indivíduo. Isso ocorreu devido a alguns indivíduos apresentarem, na primeira execução, dificuldade em realizar o exercício. Analisando as nove repetições ( $2^{\mathrm{a}}$ a $10^{\mathrm{a}}$ ) para o mesmo indivíduo, através da Anova para medidas repetidas, não se observaram variações significativas ao longo das nove repetições na potência muscular máxima ( $\mathrm{p}=$ $0,13)$. Tal fato também foi verificado ao refazer a análise com base na transformação logarítmica $(\mathrm{p}=0,38)$. Através do teste de comparações múltiplas de Bonferroni, verificou-se que os valores da primeira repetição foram significativamente menores que os obtidos nas repetições quatro e seis, não sendo observadas diferenças significativas en-

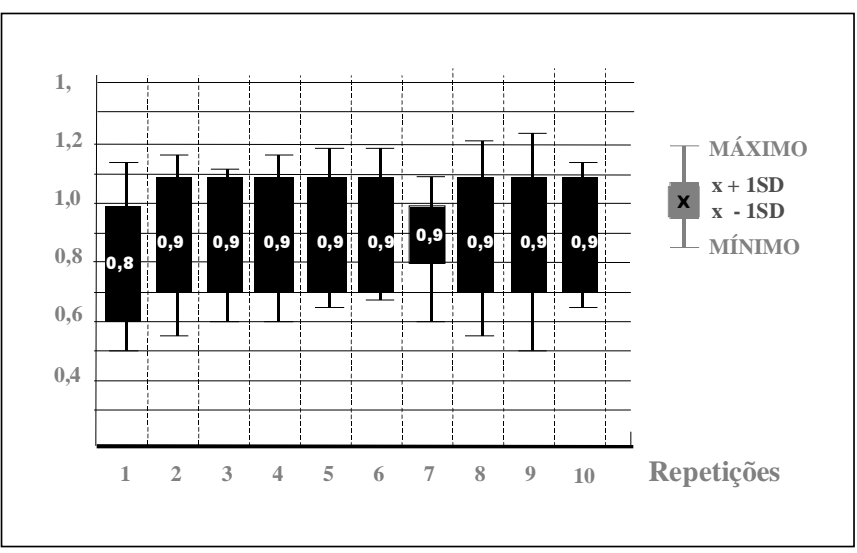

Fig. 4 - Velocidade intradias

tre os demais pares de repetições. Nas figuras 3 e 4 são ilustrados os dados referentes à potência muscular máxima e à velocidade nas dez séries realizadas no mesmo dia.

Analisando os resultados dos cinco dias para a potência muscular máxima para o mesmo indivíduo, diferenciando apenas nos dias entre as repetições, não foram observadas diferenças significativas $(p=0,69)$. Refazendo a análise dos dados após a transformação logarítmica, também não foram observadas variações significativas $(p=0,38)$.

No que diz respeito à fidedignidade interdias, as figuras 5 e 6 exibem os dados obtidos da potência muscular máxima e da velocidade nos cinco dias. Analisando as cinco medidas de velocidade, como medidas repetidas para o mesmo indivíduo, diferenciando apenas nos dias entre as repetições, verificou-se pela Anova para medidas repetidas a não existência de variação significativa ao longo dos cinco dias $(\mathrm{p}=0,66)$.

\section{DISCUSSÃO}

A literatura tem demonstrado que força e potência muscular são fundamentais tanto para a performance quanto para a saúde ${ }^{4,12,13}$. Para o treinamento desportivo, a potência muscular é uma das variáveis mais importantes ${ }^{14,15}$. Isso também é válido para as atividades cotidianas, nas quais a potência muscular desempenha um papel de grande relevância ${ }^{4,12}$. A escolha da remada em pé para o nosso estudo foi devida a sua facilidade de execução, conveniente, portanto, para indivíduos com distintos níveis de experiência motora. Contudo, um dos principais problemas associados à medida e avaliação da potência muscular parece ser sua mensuração $0^{16-20}$, principalmente no que concerne aos testes menos sofisticados que possam ser empregados em maior escala. Isso ocorre, principalmente, devido à dificuldade em quantificar com exatidão as cargas ideais e as velocidades específicas de trabalho ${ }^{4}$. 


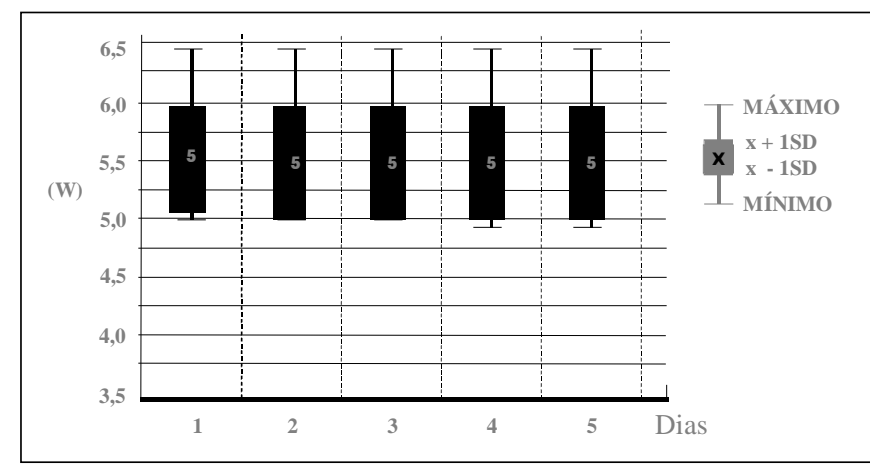

Fig. 5 - Potência muscular máxima interdias (logaritmo)

Geralmente, cargas entre 30 e $60 \%$ de 1RM são referenciadas como ideais para trabalhar exercícios de potência ${ }^{21,22}$. Por exemplo, Hoeger et al. ${ }^{23}$, em interessante estudo envolvendo levantadores de pesos, demonstraram que os exercícios de potência muscular podem chegar a $80 \%$ de uma 1 RM. Segundo esses autores, o aspecto mais importante a influenciar nas diferenças percentuais de cargas em distintos exercícios está relacionado à massa muscular envolvida. Nesse caso, quanto maior a massa muscular, menor será a potência relativa em comparação com aquela gerada por um menor grupamento. Tal afirmativa também foi confirmada por outros estudos utilizando diversos exercí$\operatorname{cios}^{17,21,24}$. No nosso estudo, utilizando a remada em pé, as cargas que geraram a potência máxima situaram-se entre 72,7 e $88,8 \%$ de 1 RM, estando estes valores ligeiramente acima do habitualmente verificado na literatura.

O que diferencia nos exercícios de fortalecimento a força e a potência musculares é a relação de velocidade para execução do movimento ${ }^{21,25,26}$. Nesse caso, o treinamento voltado para aprimoramento da força tem sido usualmente feito com velocidades baixas e cargas relativamente altas, enquanto a potência se relaciona com execuções rápidas e, conseqüentemente, cargas mais baixas ${ }^{21,24,26}$. A força geralmente está relacionada ao trabalho envolvendo cargas elevadas, sem, contudo, existir a necessidade da imposição de elevadas velocidades ao movimento. Já a potência tem sido associada a baixas cargas - ou pelo menos a cargas abaixo daquelas preconizadas para a força -, estando mais relacionada a elevadas velocidades de movimento do que à própria carga ${ }^{26}$. Tem sido preconizado que a potência muscular máxima pode ser produzida em velocidades intermediárias de movimento (aproximadamente 30\% da velocidade máxima de encurtamento) ${ }^{21-23}$. Parte da aparente controvérsia está em considerar a velocidade de 1RM como máxima, enquanto na curva clássica velocidade-carga a velocidade máxima é obtida com carga zero. Dessa forma, não se deve estranhar que nos nossos dados tivemos cargas aparentemente altas com rápidas velocidades

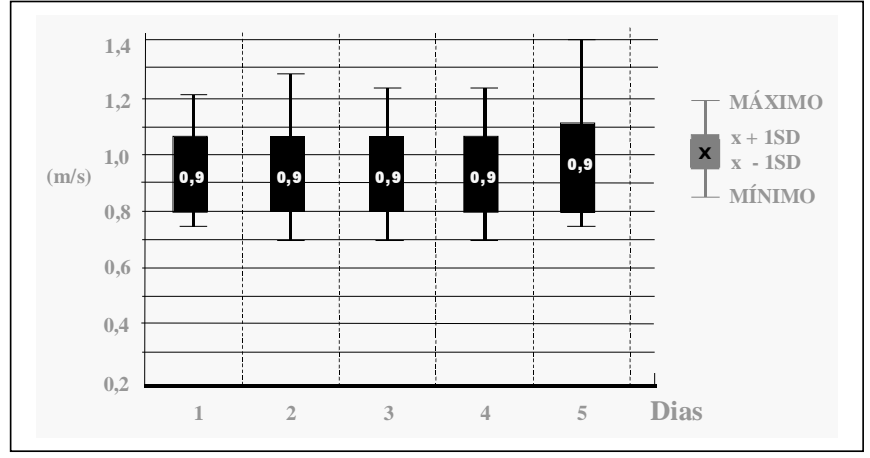

Fig. 6 - Velocidade interdias

de movimento, mostrando, na realidade, que potência verdadeiramente máxima só é alcançada com altas cargas associadas a elevadas velocidades. Um exemplo disso é que as velocidades produzidas na potência muscular máxima situam-se entre 12 e 55\% acima das velocidades obtidas na última execução do teste de 1RM.

Um dos aspectos mais difíceis de controlar no treinamento de potência é a relação ideal entre a carga e a velocidade de execução do movimento. $\mathrm{Na}$ realidade, os estudos demonstram que essa relação está interligada de maneira casual ${ }^{26,27}$. A relevância da medida da potência, realizada através do Fitrodyne, reside na facilidade para obtenção dessa relação em curto espaço de tempo. Além disso, os instrumentos destinados a medir a velocidade de movimento geralmente envolvem aparatos sofisticados, como, por exemplo, equipamentos isocinéticos ${ }^{18-20,28}$. Além do alto custo, uma das principais dificuldades em utilizar tais aparelhos reside na impossibilidade de empregar os dados em situações de campo. Exemplificando essa afirmativa, podese citar que a velocidade ideal que tende a gerar a maior potência em um dinamômetro isocinético não pode ser controlada fora do mesmo. Tal fato impede uma prescrição controlada de exercícios em situações que extrapolam o uso do equipamento. Em nossa interpretação, isso pode implicar erros grosseiros na prescrição do treinamento, já que a relação entre percentual de força e potência pode variar nas duas situações.

Outro aspecto importante a ser destacado reside na presença de dor muscular tardia, que geralmente acompanha exercícios de alta intensidade, principalmente na fase excêntrica do movimento ${ }^{29}$. Interessantemente, em nosso estudo, na medida da força através do Fitrodyne, não foi relatada dor muscular por nenhum dos voluntários nos cinco dias de medida. Outro fator que pode ter influenciado é a questão de que o treinamento com pesos objetivando potência muscular é influenciado pelo trabalho concêntrico e não pelo excêntrico, esse último mais comumente associado à dor muscular tardia ${ }^{30}$. 
No que concerne à fidedignidade dos testes de força, a literatura é rica, principalmente quanto à utilização de equipamentos mais sofisticados ${ }^{4,27,31}$. Contudo, como enfatizado por Abernethy et al. ${ }^{27}$, existe pouca consistência entre os resultados obtidos em laboratórios e sua transposição para situações de campo. Segundo esses autores, tal disparidade pode ser um problema simples de solucionar, principalmente se os pesquisadores se preocuparem em validar procedimentos que sejam mais aplicados à prescrição de exercícios. Em se tratando das medidas de potência muscular, o problema torna-se ainda maior, devido à escassez de pesquisas testando sua fidedignidade.

Ao analisar a fidedignidade de um teste deve-se considerar que seus coeficientes podem ser influenciadas por diversas variáveis, como, por exemplo, o tamanho e heterogeneidade da amostra utilizada ${ }^{27}$. Buscando avaliar a influência desse aspecto em um teste de potência muscular, determinou-se a fidedignidade inter e intradias em uma amostra composta por indivíduos com diferentes níveis de condicionamento, peso corporal, estatura e níveis de força e de potência muscular. No presente estudo, a fidedignidade nos cinco dias de testes não exerceu influência negativa nos resultados, o que permite inferir que, provavelmente, não houve efeito do aprendizado ou familiarização com o procedimento de avaliação. Todavia, um inesperado resultado significativo intradias foi observado na medida da potência muscular máxima e velocidade, para a execução da primeira repetição de alguns indivíduos. Entre os principais fatores que podem explicar esse achado, podem ser citados: inadequada voz de comando, a falta de aprendizagem adequada ao movimento, tendência de fazer mais lento do que é capaz (indivíduo inexperiente não conhece sua própria condição) ou mesmo o medo de lesionar a articulação devido às altas cargas e velocidades realizadas. Em função disso, para evitar uma contaminação dos dados, eliminou-se da análise estatística a primeira repetição no exercício selecionado para cada indivíduo, o que teria sido pro-

\section{REFERÊNCIAS}

1. Araújo DSMS, Araújo CGS. Aptidão física, atividade física e saúde. Rev Bras Med Esporte 2000;6:194-3.

2. Paffenbarger RS, Lee M. Physical activity and fitness for health and longevity. Res Q 1996;67:11-28.

3. Pollock ML, Gaesser GA, Butcher JD, Després JD, Dishman RK, Franklin BA, et al. The recommended quantity and quality of exercise for developing and maintaining cardiorespiratory and muscular fitness, and flexibility in healthy adults. Med Sci Sports Exerc 1998;30:975-91.

4. Thompson CJ, Bembem MG. Reliability and comparability of the accelerometer as a measure of muscular power. Med Sci Sports Exerc 1999; 31:897-902.

5. Feigenbaum MS, Pollock ML. Strength training - Rationale for current guidelines for adult fitness program. Phys Sportsmed 1997;25:44-64. vavelmente incorreto se a primeira repetição fosse a melhor. Como visto nas figuras 3 e 4 , a avaliação da potência muscular máxima e velocidade entre a segunda e a décima repetições não apresentou diferença significativa, demonstrando que a medida da potência muscular usando carga previamente individualizada e capaz de gerar a maior potência dentro da curva potência-carga no exercício de remada em pé, utilizando o Fitrodyne, apresenta alta fidedignidade, além de constituir-se em uma forma simples e prática, para medida da potência muscular. Outro aspecto importante a ser destacado reside na facilidade para transporte e adaptação do equipamento em diversos exercícios, fazendo com que o mesmo possa ser empregado em grandes massas populacionais.

Quando é necessário repetir medidas de força em dias consecutivos, uma das principais desvantagens dos testes isocinéticos, isométricos e dinâmicos (1RM), usualmente empregados, consiste na necessidade de recuperação, podendo chegar até três dias devido à intensidade de esforço imposta $^{27}$. No caso do teste de potência realizado pelo $\mathrm{Fi}$ trodyne, não foi verificada, em nenhum avaliado, dor muscular entre os dias de teste, fato confirmado pelos relatos dos praticantes e corroborado pela manutenção das cargas e velocidades de movimentos nos diversos dias de testes. Tal fato assume um papel de grande relevância, principalmente ao considerar as possibilidades de treinamento com elevadas cargas, similares àquelas verificadas nos testes.

Em conclusão, pode-se assumir que a medida da potência muscular pelo teste descrito usando o Fitrodyne apresenta alta fidedignidade inter e intradias, podendo ser utilizada como uma estratégia dentro de uma avaliação da função muscular. Todavia, é possível que, ao serem considerados diferentes grupamentos musculares, a fidedignidade dos testes possa variar bastante ${ }^{32}$. Nesse caso, seria interessante determinar a fidedignidade do teste de potência muscular pelo Fitrodyne em diferentes grupamentos musculares ou exercícios.
6. American College of Sports Medicine. ACSM's guidelines for exercise testing and prescription. $6^{\text {th }}$ ed. Philadelphia: Lippincott Williams \& Wilkins, 2000.

7. Harman E. Strength and power: a definition of terms. J Nat Strength Cond Assoc 1993;15:18-20.

8. Shepard RJ. PAR-Q, Canadian home fitness test and exercise screening alternatives. Sports Med 1988;5:185-95.

9. De Lorme TL, Watkins AL. Technics of progressive resistance exercise. Arch Phys Med 1948;29:263-73.

10. Gordon CC, Chunlea WC, Roche AF. Stature, recumbent length, and weight. In: Lohman TG, Roche AF, Martorell R, editors. Anthropometric standardization reference manual. Champaign: Human Kinetics, 1988; 3-8. 
11. Rosenthal MD. Shoulder savers: alterations of traditional exercises. Nat Strength Cond Assoc 1997;2:7-10.

12. Friedrich MJ. Women, exercise, and aging. JAMA 2001;285:1429-31.

13. Foldvari M, Clark M, Laviolette LC, Bernstein MA, Kaliton D, Castaneda $\mathrm{C}$, et al. Association of muscle power with functional status in community-dwelling elderly women. J Gerontol A Biol Sci Med Sci 2000; 55:192-9.

14. Kraemer WJ, Fleck SJ, Deschenes M, Evans WJ. Strength and power training: physiological mechanisms of adaptation. Exerc Sport Sci Rev 1996;24:363-97.

15. Kanehisa H, Miyashita M. Specificity of velocity in strength training. Eur J Appl Phys 1983;52:104-6.

16. Murphy AI, Wilson GJ, Pryor JF. The use of inertial force mass relationship in the prediction of dynamic human performance. Eur J Appl Physiol 1994;69:250-7.

17. Agre JC, Magness JL, Hull SZ. Strength testing with a portable dynamometer: reliability for upper and lower extremities. Arch Phys Med Rehab 1987;68:454-8.

18. Coyle EF, Feiring DC, Rotkes TC, Cote RW, Roby FB, Lee W, Wilmore JH. Specificity of power improvements through slow and fast isokinetic training. J Appl Phys 1981;51:1437-42.

19. Kannus P. Isokinetic evaluation of muscular performance: implications for muscle testing and rehabilitation. J Sports Med 1994;15:11-8.

20. Kanehisa H, Miyashita M. Effect of isometric and isokinetic muscle training on static strength and dynamic power. Eur J Appl Physiol 1983;50: 365-71.

21. Komi PV. Strength and power in sport. Oxford: Blackwell Science, 1995; 249-63

22. Cronin JB, McNair PJ, Marshall RN. The role of maximal strength and load on initial power production. Med Sci Sports Exerc 2000;32:1763-9.
23. Hoeger WWK, Hopkins DR, Barette SL. Relationship between repetitions and selected percentages of one repetition maximum: a comparison between untrained and trained males and females. J Appl Sport Sci Res 1990;4:47-54.

24. Fleck SJ, Kraemer WJ. Designing resistance training programs. $2^{\text {nd }}$ ed. Champaign: Human Kinetics 1997;137-28.

25. Hakkinen K, Alen M, Komi PV. Effect of explosive type strength training on isometric force and relaxation-time, electromyographic and muscle fibre characteristics of leg extensor muscle. Acta Phys Scan 1985; 125:587-600.

26. Maud PJ, Foster C. Physiological assessment of human fitness. Champaign: Human Kinetics, 1995;115-32.

27. Abernethy P, Wilson G, Logan P. Strength and power assessment: issues, controversies and challenges. Sports Med 1995;19:401-17.

28. Komi PV, Linnamo V, Silventoinen P, Sillanpää M. Force and EMG power spectrum during eccentric and concentric actions. Med Sci Sports Exerc 2000;32:1757-62.

29. Smith LL, Fulmer MG, Holbert D, McCammon MR, Hourmard JA, Frazer DD, et al. The impact of a repeated bout of eccentric exercise on muscular strength, muscle soroness and creatine kinase. J Sports Med 1994;28:267-71.

30. Dolezal BA, Potteiger JA, Jacobsen DJ, Benedict SH. Muscle damage and resting metabolic rate after acute resistance exercise with an eccentric overload. Med Sci Sports Exerc 2000;32:1202-7.

31. Dolny DG, Collins MG, Wilson T, Germann ML, Davis HP. Validity of lower extremity strength and power utilizing a new closed chain dynamometer. Med Sci Sports Exerc 2001;33:171-5.

32. Christ CB, Slaugter MH, Stillman RJ. Reliability of selected parameters of isometric muscle function associated with testing 3 days $\mathrm{x} 3$ trials in women. J Strength Cond Res 1994;8:65-71. 